\title{
Adaptive fuzzy self-learning controller based rotor resistance estimator for vector controlled induction motor drive
}

\author{
Moulay Rachid Douiri ${ }^{\mathrm{a}}$ And Mohamed Cherkaoui \\ Mohammadia Engineering School, Department of Electrical Engineering, Avenue Ibn Sina, B.P. 765, Agdal-Rabat, Morocco
}

Received 27 June 2013, Accepted 8 October 2014

\begin{abstract}
This paper presents an intelligent approach to identify and adapt the rotor resistance for an indirect vector controlled induction motor drive. This command is affected by rotor resistance; the variation of this parameter could distort the decoupling between flux and torque and, consequently, lead to deterioration of drive performance. To overcome this problem, a fuzzy estimator is provided to identify the real value of rotor resistance in order to obtain a vector control optimal. Then we propose a fuzzy adaptive control strategy fits into the learning methods context by modifying the consequences of fuzzy estimator. Regarding the learning algorithm, our solution envisages the use of a fuzzy inverse model, combined with a mechanism that acts based on estimator rules by modifying the consequents according to a certain criterion, so as to increase the system robustness, and avoid unnecessary oscillation in the control signal. The suggested rotor resistance identification approach has been validated by simulation study.
\end{abstract}

Key words: Adaptive control / fuzzy logic / induction motor / learning system / rotor resistance

\section{Introduction}

In indirect field control (IFOC) the rotor resistance is an important parameter which is involved in rotor flux estimation and the control law to compensate for the nonlinearity of system [1]. However, this parameter varies with machine temperature. In addition it was demonstrated that a poor estimate of this parameter affects the regulation (pursuit of flux trajectory and rotor speed) and even it can introduce oscillations [1]. This difficulty has been the main source of our motivation for this research. Several authors have contributed to estimating rotor resistance [2-6]; in which one proposes a new self-learning adaptive fuzzy controller to identify the rotor resistance.

Fuzzy logic control or fuzzy control is a nonlinear control method first proposed in 1965 by Dr. Zadeh [2]. It controls systems heuristically using a knowledge base specified by the designer, hence imitating human logic in order to perform control actions. The biggest advantage of this controller is its ability to deal with systems that are uncertain (fuzzy) due to complexity, incompleteness, disturbances, etc., which are difficult to model using conventional controllers $[7,8]$.

Each classical fuzzy logic controller contains a fuzzifier, a rule base, a fuzzy inference engine and a defuzzifier. There are many alternatives to choose from during the design process for the membership function type associated

\footnotetext{
${ }^{a}$ Corresponding author: douirirachid@hotmail.com
}

with the input and output fuzzy sets, the fuzzy inference type, the T-Norm operator, the S-Norm operator and the defuzzifier type $[8,9]$. However once the choice is made, it is final. All the calculation methods as well as the parameters are fixed during real time operation.

Adaptive fuzzy system is implemented in the framework of adaptive network architecture and equipped with a training (adaptation) algorithm with supervised learning, data are presented to the network input and the latter computes and produces outputs. The differences between these outputs and the desired outputs form errors which are corrected using the back-propagation algorithm, by applying this step several times, the error tends to decrease and the network provides a better prediction [10-12].

Our adaptation strategy fits into the learning methods context by modifying consequences of the fuzzy estimator [13-15]. This choice is inspired by the performance of these methods which are, also, very intuitive. This class of strategies is derived from the Mamdani [16] work where the concept of self-organized control has been developed. As well we will further study this category of fuzzy adaptation methods, and propose a solution to the problems detected in existing algorithms. In this way, we hope able to maximum exploit the characteristics of adaptive strategy. Regarding the learning algorithm, our solution envisages the use of a fuzzy inverse model, combined with a mechanism that acts based on estimator rules by 


\section{Abbreviations and symbols}

\begin{tabular}{|c|c|}
\hline IFOC & Indirect Field Oriented Control \\
\hline DFOC & Direct Field Oriented Control \\
\hline FOC & Field Oriented Control \\
\hline FLE & Fuzzy Logic Estimator \\
\hline FRRE & Fuzzy Rotor Resistance Estimator \\
\hline AFSLRRE & Adaptive Fuzzy Self-Learning based Rotor Resistance Estimator \\
\hline$v_{s d}, v_{s q}$ & Stator voltages in $d-q$ axes $[\mathrm{V}]$ \\
\hline$i_{s d}, i_{s q}$ & Stator currents in $d-q$ axes $[\mathrm{A}]$ \\
\hline$i_{r d}, i_{r q}$ & Rotor currents in $d-q$ axes $[\mathrm{A}]$ \\
\hline$\lambda_{r d}, \lambda_{r q}$ & Rotor flux components in $d-q$ axes [Wb] \\
\hline$R_{s}, R_{r}$ & Stator and rotor resistances $[\Omega]$ \\
\hline$L_{m}, L_{s}, L_{r}$ & Mutual, stator, and rotor inductances $[\mathrm{H}]$ \\
\hline$T_{e}, T_{l}$ & Electromagnetic torque, mechanical loads [N.m] \\
\hline$\omega_{e}, \omega_{r}, \omega_{s l}$ & Synchronous, rotor, and slip frequencies [rad/s] \\
\hline$\theta_{e}, \theta_{r}, \theta_{s l}$ & Synchronous, rotor, and slip angles [rad] \\
\hline$f$ & Damping coefficient [N.m.s] \\
\hline$\tau_{r}$ & Rotor time constant $[\mathrm{s}]$ \\
\hline$J$ & Inertia moment $\left[\mathrm{kg} \cdot \mathrm{m}^{2}\right]$ \\
\hline$n_{p}$ & Number of pole pairs \\
\hline$P_{n}$ & Rated power $[\mathrm{kW}]$ \\
\hline$R_{-e s t}$ & Estimated rotor resistance \\
\hline$\Phi, \Phi_{a c t}, \Phi_{e s t}, \Phi_{m}$ & Function, actual function, estimation function, and function of reference model output \\
\hline$\mu()$. & Membership function \\
\hline$E_{\Phi}, \Delta E_{\Phi}, \Delta R_{r}$ & Error function, error change function, and rotor resistance change \\
\hline$E_{\Phi m}, \Delta E_{\Phi m}, \Delta R_{r 2}$ & $\begin{array}{l}\text { Error function, error change function, and rotor resistance change } \\
\text { in the output variable adjustment mechanism }\end{array}$ \\
\hline$\varepsilon_{\Phi}, \Delta \varepsilon_{\Phi}, \Delta r_{r}$ & Error function normalized, error change function normalized, and rotor resistance change normalized \\
\hline$\varepsilon_{\Phi m}, \Delta \varepsilon_{\Phi m}, \Delta r_{r 2}$ & $\begin{array}{l}\text { Error function normalized, error change function normalized, and rotor resistance change normalized } \\
\text { in the output variable adjustment mechanism }\end{array}$ \\
\hline$G_{E}, G_{\Delta E}, G_{\Delta r}$ & Gain error, gain error change, and gain output variable change \\
\hline$G_{E m}, G_{\Delta E m}, G_{\Delta r 2}$ & $\begin{array}{l}\text { Gain error, gain error change, and gain output variable change in the output variable } \\
\text { adjustment mechanism }\end{array}$ \\
\hline$F_{t}$ & Fitness function \\
\hline$C_{i}$ & Consequent fuzzy sets of output \\
\hline$z^{-1}$ & Unit delay \\
\hline$s$ & Laplace variable \\
\hline
\end{tabular}

modifying the consequents, according to a certain criterion, so as to increase the system robustness, and avoid unnecessary oscillation in the control signal. Adaptation strategy must guarantee high rotor resistance estimation. On the other hand, it is desirable that the adaptation law can be obtained from a simplified model of induction machine and the electric actuator. From standpoint of topology control, we propose a fuzzy estimator placed on the main chain of control loop, and a fuzzy adaptation mechanism that makes decisions based on a reference model, in order to modify the estimator base rules.

This paper is organized as follows: the principle of indirect field oriented control is presented in second section, the structure of fuzzy logic rotor resistance estimator is explained in third section, the design of adaptive fuzzy self-learning based rotor resistance estimator is developed in section four; the fifth section is devoted to illustrate the simulation performance of this control strategy, finally, section six presents our conclusions.

\section{Indirect field oriented control}

The idea of FOC is to assume an axis rotating with synchronous frequency (i.e. rotate at same frequency of rotating field). Then by alignment of flux vector along the $d$-axis of synchronously rotating reference frame the other flux component will be zero. Hence, if flux is kept constant $\left(\lambda_{r d}\right)$ then torque will be proportional to the $q$-axis current component $\left(i_{q}\right)$. Figure 1 shows the vector diagram for indirect field oriented control. 


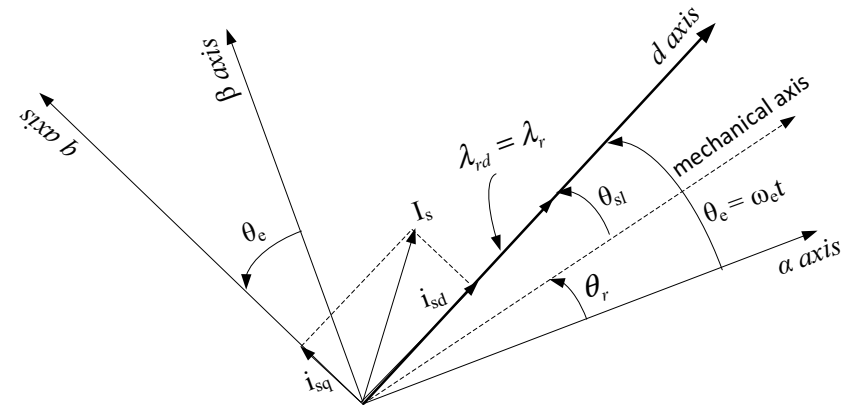

Fig. 1. Vector diagram for indirect field oriented control.

At any instant, $d$ electrical axis is in angular position $\theta_{e}$ relative to $\alpha$ axis. The angle $\theta_{e}$ is the result of sum of both rotor angular and slip angular positions, as follows:

$$
\left\{\begin{array}{l}
\theta_{e}=\theta_{r}+\theta_{s l} \\
\omega_{e} t=\omega_{r} t+\omega_{s l} t=\left(\omega_{r}+\omega_{s l}\right) t
\end{array}\right.
$$

The rotor dynamics are given by following equations:

$$
\begin{aligned}
\frac{\mathrm{d} \lambda_{r}}{\mathrm{~d} t} & =\frac{L_{m}}{\tau_{r}} i_{s d}-\frac{1}{\tau_{r}} \lambda_{r} \\
\frac{\mathrm{d} \omega_{r}}{\mathrm{~d} t} & =\frac{3 n_{p}^{2} L_{m}}{2 J L_{r}} \lambda_{r} i_{s q}-\frac{f}{J} \omega_{r}-\frac{n_{p}}{J} T_{l} \\
T_{e} & =\frac{3 n_{p} L_{m} \lambda_{r}}{2 L_{r}} i_{s q} \\
\theta_{e} & =\int \omega_{e} \mathrm{~d} t=\int\left(\omega_{r}+\frac{R_{r} L_{m} i_{s q}}{L_{r} \lambda_{r}}\right) \mathrm{d} t
\end{aligned}
$$

The rotor flux magnitude is related to the $d$-axis stator current by a first-order differential equation; thus, it can be controlled by controlling the direct axis stator current. Under steady-state operation rotor flux is constant, so equation (2) becomes:

$$
\lambda_{r}=L_{m} i_{s d}
$$

Indirect vector control can be calculated as follow:

$$
\begin{aligned}
i_{s d}^{*} & =\frac{\lambda_{r}^{*}}{L_{m}} \\
i_{s q}^{*} & =\frac{2 L_{r} T_{e}^{*}}{3 n_{p} L_{m} \lambda_{r}^{*}} \\
\omega_{s l}^{*} & =\frac{R_{r} L_{m} i_{s q}^{*}}{L_{r} \lambda_{r}^{*}} \\
\theta_{e}^{*} & =\int \omega_{e}^{*} \mathrm{~d} t=\int\left(\omega_{r}+\omega_{s l}^{*}\right) \mathrm{d} t
\end{aligned}
$$

\section{Fuzzy rotor resistance estimator (FRRE)}

The estimator input variables should wear explicitly or implicitly information related to the resistance variation [2]. We find that torque could be the candidate. We can estimate actual torque from stator flux. This method is simple but it is not recommended for low-speed operation since in this region it is very difficult to estimate exactly the stator flux $[17,18]$.

We will use a function $\Phi$, which is a modification of function used in equation (3):

$$
\Phi=\frac{1}{\omega_{e}}\left(i_{q s} \frac{\mathrm{d} \lambda_{\mathrm{d} r}}{\mathrm{~d} t}-i_{\mathrm{d} s} \frac{\mathrm{d} \lambda_{q r}}{\mathrm{~d} t}\right)
$$

As the resistance variation with temperature is very slow, we can estimate in steady state. We can demonstrate that for steady state the function $\Phi$ can be calculated as follows:

$$
\Phi=-i_{d s} \lambda_{d r}=-i_{d s} \hat{\lambda}_{d r}=\Phi_{e s t}
$$

where $\hat{\lambda}_{d r}$ is the estimated flux of $d$-axis.

The actual value of function $\Phi$ is calculated:

$$
\Phi_{a c t}=\frac{L_{r}}{L_{m}}\left[\frac{1}{\omega_{e}}\left(v_{d s} i_{q s}-v_{q s} i_{d s}\right)+L_{s} \sigma\left(i_{d s}^{2}+i_{q s}^{2}\right)\right]
$$

In equation (13) the function $\Phi$ is calculated from stator voltage and current. The rotor voltage will be available to our estimation algorithm. Figure 2 shows the configuration of rotor resistance estimation. The functions $\Phi_{\text {est }}$ and $\Phi_{\text {act }}$ are first calculated.

The error between $\Phi_{\text {est }}$ and $\Phi_{a c t}$ and its derivation are the estimator inputs $\Delta E_{\Phi}(k)$, they are then calculated as follows:

$$
\begin{aligned}
E_{\Phi}(k) & =\Phi_{e s t}(k)-\Phi_{a c t}(k) \\
\Delta E_{\Phi}(k) & =E_{\Phi}(k)-E_{\Phi}(k-1)
\end{aligned}
$$

The internal structure of estimator involves three steps: fuzzification, inference and defuzzification. The signals $\varepsilon_{\Phi}(k)$ and $\Delta \varepsilon_{\Phi}(k)$ are normalized and deducted from the signals $E_{\Phi}(k)$ and $\Delta E_{\Phi}(k)$ by multiplying by factor $G_{E}$ and $G_{\Delta E}$.

Triangular shaped membership functions, Z-shaped membership functions, and S-shaped membership functions were used for all inputs and outputs of the fuzzy controller. The membership functions are uniformly distributed over the inputs and outputs dynamic range (Fig. 3). The rule-based fuzzy estimator is built based on process information. The basic structure of such a rulebase is shown in Table 1.

The $i$ th rule $R_{i}$ can be expressed as:

$$
\begin{array}{r}
R_{i}: \quad \text { if } \varepsilon_{\Phi} \text { is } A_{i}, \quad \text { and } \Delta \varepsilon_{\Phi} \text { is } B_{i}, \\
\text { then } \Delta r_{r} \text { is } N_{i}
\end{array}
$$

where $A_{i}$ and $B_{i}$ denote the fuzzy subsets and $N_{i}$ is a fuzzy singleton set.

The inference method used in this paper is Mamdani's procedure based on min-max decision [11]. The firing strength $\chi_{i}$, for $i$ th rule is given by:

$$
\chi_{i}=\min \left(\mu_{A_{i}}\left(\varepsilon_{\Phi}\right), \mu_{B_{i}}\left(\Delta \varepsilon_{\Phi}\right)\right)
$$

By fuzzy reasoning, Mamdani's minimum procedure gives:

$$
\mu_{N_{i}}^{\prime}\left(\Delta r_{r}\right)=\min \left(\chi_{i}, \mu_{N_{i}}\left(\Delta r_{r}\right)\right)
$$




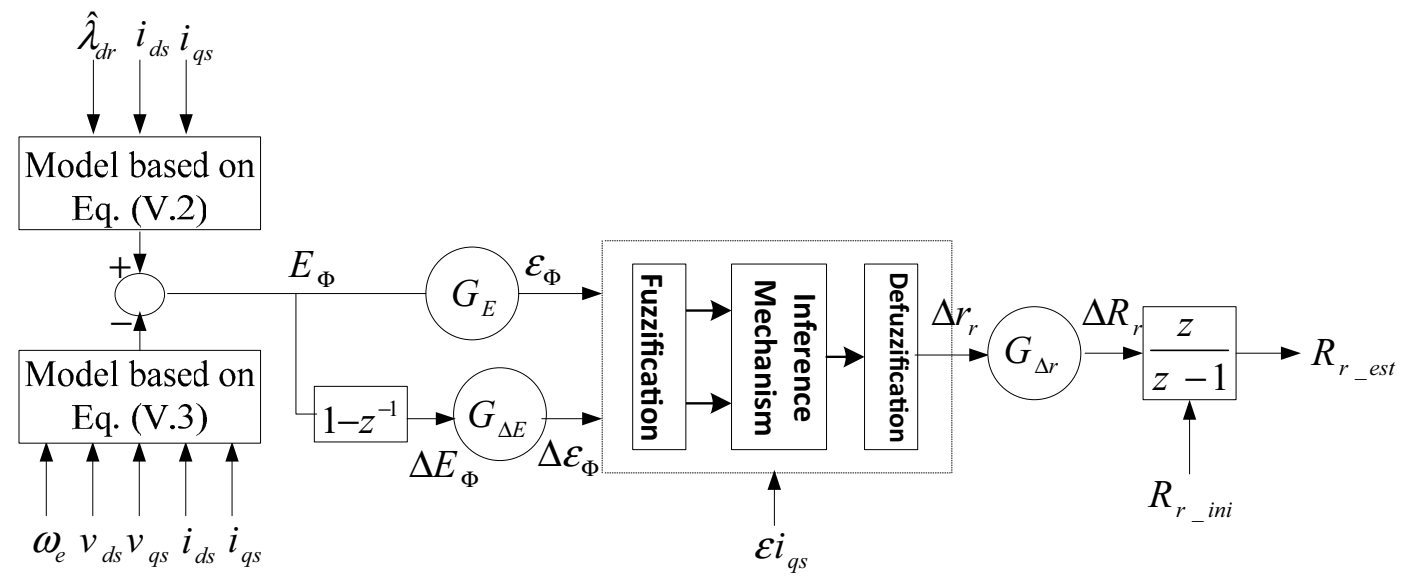

Fig. 2. Structure of the fuzzy rotor resistance estimator.

Table 1. Rule base for fuzzy rotor resistance identification.

\begin{tabular}{|c|c|c|c|c|c|c|c|c|}
\hline & & \multicolumn{7}{|c|}{$\Delta \varepsilon_{\Phi}$} \\
\hline & & $\overline{\mathrm{NB}}$ & $\mathrm{NM}$ & $\mathrm{NS}$ & $\mathrm{Z}$ & $\overline{\mathrm{PS}}$ & $\overline{\mathrm{PM}}$ & $\overline{\mathrm{PB}}$ \\
\hline \multirow{7}{*}{$\varepsilon_{\Phi}$} & NB & $\mathrm{NB}$ & $\mathrm{NM}$ & $\mathrm{NM}$ & $\mathrm{NS}$ & $\mathrm{NS}$ & NS & $\mathrm{Z}$ \\
\hline & $\mathrm{NM}$ & $\mathrm{NM}$ & NM & NS & NS & NS & Z & PS \\
\hline & NS & NM & $\mathrm{NM}$ & NS & $\mathrm{NS}$ & $\mathrm{Z}$ & PS & PM \\
\hline & $\mathrm{Z}$ & NB & NM & NS & Z & PS & PM & $\mathrm{PM}$ \\
\hline & PS & NS & NS & $\mathrm{Z}$ & PS & PS & PM & $\mathrm{PM}$ \\
\hline & PM & NS & $\mathrm{Z}$ & PS & PS & PS & $\mathrm{PM}$ & $\mathrm{PM}$ \\
\hline & PB & Z & PS & PS & $\mathrm{PM}$ & $\mathrm{PM}$ & PB & $\mathrm{PB}$ \\
\hline
\end{tabular}

where $\mu_{A}, \mu_{B}$, and $\mu_{N}$ are membership functions of sets $A, B$, and $N$ of the variables $\varepsilon_{\Phi}, \Delta \varepsilon_{\Phi}$, and $\Delta r_{r}$, respectively.

Thus, the membership function $\mu_{N}$ of the output $n$ is given by:

$$
\mu_{N}\left(\Delta r_{r}\right)=\max _{i=1}^{49}\left(\mu_{N_{i}}^{\prime}\left(\Delta r_{r}\right)\right)
$$

The rules table contains 49 rules $(7 \times 7)$ as shown in Table 1 .

The estimated value of the resistance increment is obtained by multiplying the estimator output $\Delta r_{r}(k)$ by the gain $G_{\Delta r}$. Resistance is finally integration increment:

$$
R_{r_{-} e s t}(k)=R_{r}(k-1)+G_{\Delta r} \cdot \Delta r_{r}(k)
$$

Note that the initial value of the resistance $R_{r}$ is its nominal value.

This resistance is estimated to be used in indirect vector control algorithm to ensure optimal performance.

The fuzzy sets are characterized by standard designations: NB (negative big), NM (negative medium), NS (negative small), $Z$ (zero), PS (positive small), PM (positive medium) and $\mathrm{PB}$ (positive big).

As the time constant of resistance variation as a function of temperature is much greater than motor electric time constant, we can estimate the resistance in steady state, where there is no load variation or change control signal. This ensures that signal variations $E_{\Phi}(k)$ and $\Delta E_{\Phi}(k)$ are caused by $R_{r}$ variation only.

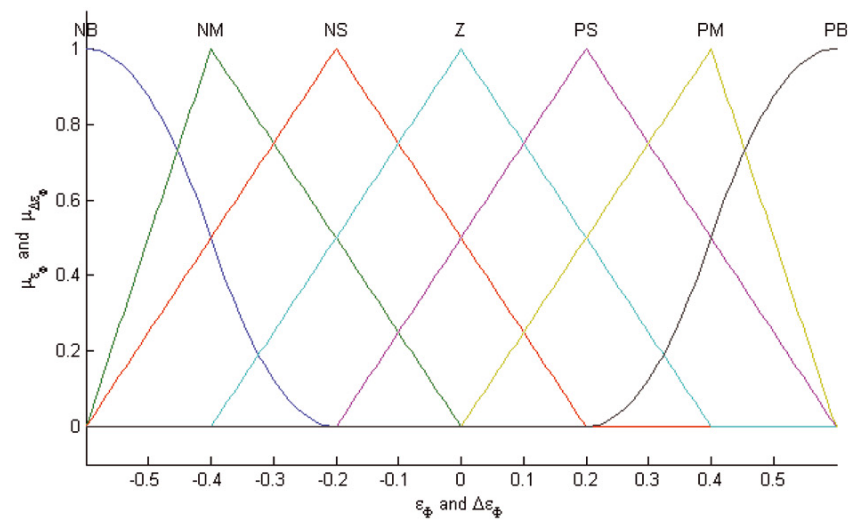

(a)

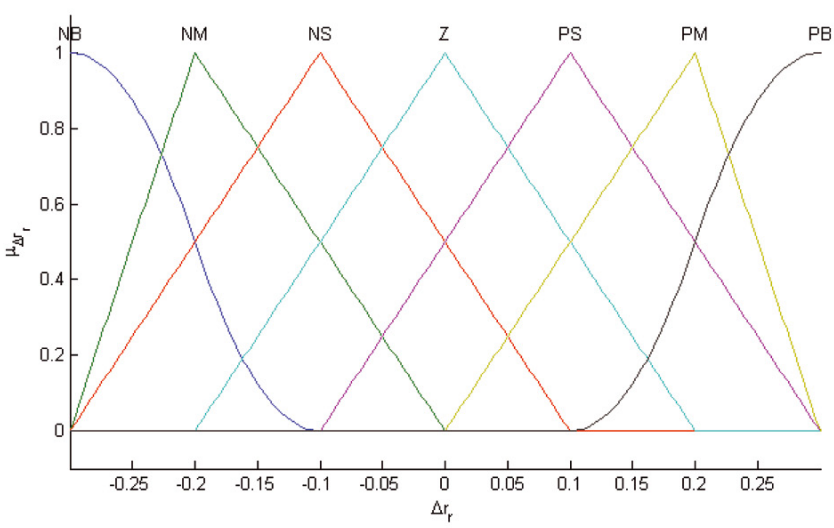

(b)

Fig. 3. Input and output membership functions; (a) $\varepsilon_{\Phi}$ and $\Delta \varepsilon_{\Phi},(\mathrm{b}) \Delta r_{r}$.

\section{Adaptive fuzzy self-learning based rotor resistance estimator (AFSLRRE)}

The structure of fuzzy model reference control is shown in Figure 4. In this control scheme the system (the plant together with the controller) is asked to follow certain performances given by a reference model. We define 


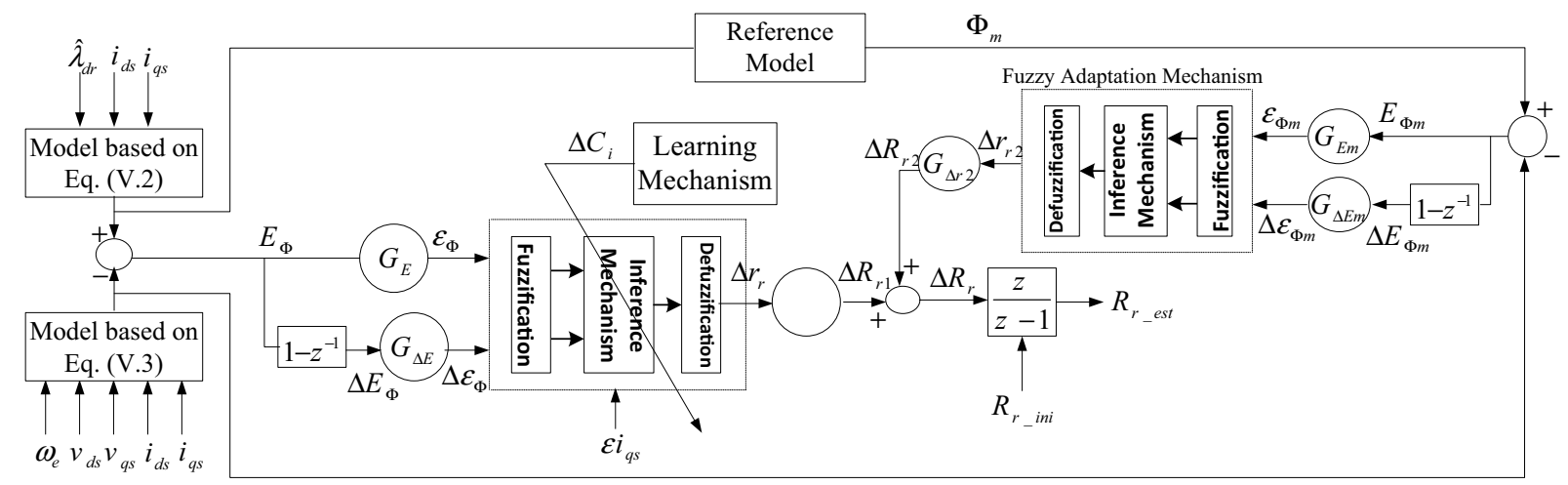

Fig. 4. Block diagram of a new adaptive fuzzy self learning for rotor resistance estimator.

the pursuit error and its variation:

$$
\begin{aligned}
E_{\Phi m}(k) & =\Phi_{m}(k)-\Phi_{a c t}(k) \\
\Delta E_{\Phi m}(k) & =E_{\Phi m}(k)-E_{\Phi m}(k-1)
\end{aligned}
$$

These input variables are processed by adaptation system using fuzzy rules to produce a signal adaptation $\Delta R_{r 2}$ which is added to the output of FLE direct:

$$
\Delta R_{r}(k)=\Delta R_{r 1}(k)+\Delta R_{r 2}(k)
$$

Estimated resistance is obtained by integrating the signal:

$$
R_{r_{-} e s t}(k)=R_{r_{-} e s t}(k-1)+\left(\Delta R_{r 1}(k)+\Delta R_{r 2}(k)\right)
$$

Normally, the reference model is a linear 1st or 2nd order system. In this work a 2nd order system is used as a reference model given by:

$$
\frac{\Phi_{a c t}(s)}{\Phi_{\text {est }}(s)}=\frac{\omega_{n}^{2}}{s^{2}+2 \xi \omega_{n}+\omega_{n}^{2}}
$$

where $\omega_{n}$ is the desired natural frequency and $\xi$ is the desired damping ratio.

It is possible to select the reference model with a higher order to match a process of higher dynamics. However, it is easier to describe the desired performance using a 2 nd order system by selecting both $\omega_{n}$ and $\xi$.

The adaptation mechanism is responsible for forming the rules at the rule-base of fuzzy estimator. These rules describe the non-linear control surface that compensates and linearizes the overall system to match the reference model. It is also responsible of adapting the control surface to compensate according to the process varying parameters. The inputs of the main fuzzy controller are as equations (21) and (22). Uniformly distributed triangle membership functions are used for this work for the controller inputs and output for the main fuzzy controller. We choose min-max inference method; the defuzzification process employs the center of gravity method. The structure of the rule-base is identical to the one used for the main fuzzy controller in the FRRE for simplicity and is shown in Table 1. If more information about the process is available it is possible to describe it in the rule-base of the fuzzy controller.
The objective of the adaptation loop is to minimize a cost function capable of "measuring" the performance of system:

$$
F_{t}(k)=\rho_{E_{\Phi}} \frac{E_{\Phi m}^{2}(k)}{2}+\rho_{\Delta E_{\Phi}} \frac{\Delta E_{\Phi m}^{2}(k)}{2}, \rho_{E_{\Phi}}, \rho_{\Delta E_{\Phi}} \succ 0
$$

To minimize the cost function $F_{t}$, must change the consequent in the direction of the negative gradient of $F_{t}$. It should be noted, however, that only consequent rules corresponding active will be affected, i.e., the rules which the activation degree is different from zero. The parameters updating is performed according to the expression:

$$
C_{i}(k)_{n e w}=C_{i}(k)_{o l d}+\Delta C_{i}(k)
$$

In the learning process, a certain delay is considered, so that the control signal $\Delta R_{r}$ will have a dominant effect that after the next $p$ calculation steps, where $p \geq n$. According to the gradient method, consequent variations will be defined as:

$$
\Delta C_{i}(k) \propto-\frac{\partial F_{t}(k+p)}{\partial C_{i}(k)}=-\frac{\partial F_{t}(k+p)}{\partial \Delta R_{r}(k)} \frac{\partial \Delta R_{r}(k)}{\partial C_{i}(k)}
$$

Using equations (21), (22) and (26), we obtain:

$$
\begin{gathered}
\frac{\partial F_{t}(k+p)}{\partial \Delta R_{r}(k)}=-\rho_{E_{\Phi}} E_{\Phi m}(k+p) \frac{\partial \Phi_{a c t}(k+p)}{\partial \Delta R_{r}(k)} \\
-\rho_{\Delta E_{\Phi}} \Delta E_{\Phi m}(k+p) \frac{\partial\left(\Phi_{a c t}(k+p)-\Phi_{a c t}(k+p-1)\right)}{\partial \Delta R_{r}(k)}
\end{gathered}
$$

However,

$$
u^{*}=\frac{\sum_{i=1}^{N} \mu_{i} c_{i}}{\sum_{i=1}^{N} \mu_{i}}, u=K_{u} u^{*}
$$

After equation (30)

$$
\frac{\partial \Delta R_{r}(k)}{\partial C_{i}(k)}=K_{u} \frac{\mu_{i}}{\sum_{l=1}^{N} \mu_{i}}
$$


Thus, equation (28) becomes:

$$
\begin{gathered}
\Delta C_{i}(k) \propto\left[\rho_{E_{\Phi}} E_{\Phi m}(k+p) \frac{\partial \Phi_{a c t}(k+p)}{\partial \Delta R_{r}(k)}\right. \\
\left.+\rho_{\Delta E_{\Phi}} \Delta E_{\Phi m}(k+p) \frac{\partial\left(\Phi_{a c t}(k+p)-\Phi_{a c t}(k+p-1)\right)}{\partial \Delta R_{r}(k)}\right] \\
\times K_{u} \frac{\mu_{i}}{\sum_{l=1}^{N} \mu_{l}}
\end{gathered}
$$

The partial derivatives in equation (33) can be expressed by positive constants, provided they are positive at least not from the future $p$ steps. The delay of learning $p$ is a project parameter, determined by taking into account the process order and possible unstable zeros. In this way, the partial derivatives will be included in the learning gains, and the approximation of equation (32) is written as:

$$
\begin{aligned}
\Delta C_{i}(k)= & \left(\delta_{E_{\Phi}} E_{\Phi m}(k+p)\right. \\
& \left.+\delta_{\Delta E_{\Phi}} \Delta E_{\Phi m}(k+p)\right) K_{u} \frac{\mu_{i}}{\sum_{l=1}^{N} \mu_{l}}
\end{aligned}
$$

Given the foregoing, the learning algorithm is based, essentially on an iterative procedure offline. Defining reference signals of interest in learning, the iteration loop is executed until the corrector learns to act on the process, so that the pursuit of objectives is achieved. Figure 4 shows a block diagram of robust learning fuzzy rotor resistance estimator.

\section{Numerical results}

In order to test the validity of the new proposed approach algorithm, a numerical analysis is carried out, which has been simulated using the MATLAB/Slmulink environment. The induction motor parameters are listed as follows:

$P_{n}=3 \mathrm{~kW}, V_{n}=230 \mathrm{~V}, R_{s}=2.89 \Omega, R_{r}=2.39 \Omega$, $L_{s}=0.225 \mathrm{H}, L_{r}=0.220 \mathrm{H}, L_{m}=0.214 \mathrm{H}, J=$ $0.2 \mathrm{~kg} . \mathrm{m}^{2}, n_{p}=2$.

The rotor resistance is changed abruptly during steady state operation of the drive. Its value is increased from the nominal value of $2.39 \Omega\left(R_{r}=100 \% R_{r, n}\right)$ to $3.585 \Omega$ $\left(R_{r}=150 \% R_{r, n}\right)$ at $0.2 \mathrm{~s}$, and then decreased to $2.987 \Omega$ $\left(R_{r}=125 \% R_{r, n}\right)$ at $0.4 \mathrm{~s}$, and then decreased to 1.195 $\left(R_{r}=50 \% R_{r, n}\right) \Omega$ at $0.6 \mathrm{~s}$.

Figures $5 \mathrm{a}$ and $5 \mathrm{a}^{\prime}$ present the estimation and error estimation results of rotor resistance using FRRE and AFSLRRE. These two figures show a good operation of the AFSLRRE which does not depend, again, the initial value of rotor resistance chosen in the algorithm. This is very important since in reality we do not know the exact value of resistance when the estimation algorithm starts. The convergence of this method is thus confirmed. In reality, the actual rotor resistance varies much more slowly, this means that the estimated rotor resistance can better monitor the actual rotor resistance.
Figure 5b shows the speed response of both techniques, when the rotor resistance changes abruptly. The rotor speed with AFSLRRE strategy drops to $199.9 \mathrm{rad} / \mathrm{s}$ at $R_{r}=150 \% R_{r, n}$ and rises to $200.1 \mathrm{rad} / \mathrm{s}$ at at $R_{r}=$ $125 \% R_{r, n}$ and $200.2 \mathrm{rad} / \mathrm{s}$ at $R_{r}=50 \% R_{r, n}$ and then is adjusted back to its demanded value in $0.8 \mathrm{~ms}, 0.6 \mathrm{~ms}$ and $0.9 \mathrm{~ms}$ respectively, with a steady state error of $0.003 \%$, $0.002 \%$ and $0.005 \%$ respectively. while the FRRE strategy drops to $197.3 \mathrm{rad} / \mathrm{s}$ at $R_{r}=150 \% R_{\mathrm{r}, \mathrm{n}}$ and rises to $202.7 \mathrm{rad} / \mathrm{s}$ at $R_{r}=125 \% R_{r, n}$ and $202.9 \mathrm{rad} / \mathrm{s}$ at $R_{r}=50 \% R_{r, n}$ and then is adjusted back to its demanded value in $0.05 \mathrm{~s}, 0.03 \mathrm{~s}$, and $0.06 \mathrm{~s}$ respectively, with a steady state error of $0.02 \%, 0.01 \%$ and $0.05 \%$, respectively. AFSLRRE shows more robustness against rotor resistance variation as well as high disturbance rejection capability compared to FRRE.

The curves of flux and torque (Figs. $5 \mathrm{c}$ and $5 \mathrm{~d}$ ) remain at their respective set-points despite the disturbance of $100 \%, 150 \%, 125 \%$ and $50 \%$ applied to the rotor resistance. This proves that the adaptation process of this parameter is effectively realized and that decoupling is maintained. Indexical variations were used here in order to verify the dynamic performance estimation scheme. However, in practice the rotor resistance varies exponentially with the motor heating. From Table 2, we have summarized the simulation results under the effect of the rotor resistance variations.

We can therefore observe the little operations involved in this estimator, which would result in an execution time fairly rapid especially when these operations are implemented on processors optimized for calculations such as is the case of digital signal processors (Tab. 3).

By comparing the results, one can say that the AFSLRRE provides excellent dynamic performance to an induction motor drive than the FRRE that appears in the literature. Insensitivity to the drive parameter variations and working conditions can be thus obtained.

\section{Conclusion}

The expected results of this research were primarily to improve the drive performance addressed by conventional methods of indirect rotor flux orientation. This improvement should be achieved by developing a new strategy indirect more robust with respect to the parameter variation. The basic idea of this new control structure is the online correction of rotor resistance variation to maintain the decoupling controller in perfect agreement with the actual conditions of motor operation. The topology control is constituted by a fuzzy estimator whose rule base is synthesized by the adaptation mechanism. We have for goal to attack the problem of modification of consequents fuzzy estimator, so that the algorithm adaptation and updating of these parameters has a regulatory mechanism to avoid the generation of not bounded values. Rule base will be synthesized and coherent with the objectives solicited control. Taking into account the results obtained by simulation, we can affirm that AFSLRRE has better dynamic performance than FRRE presented 


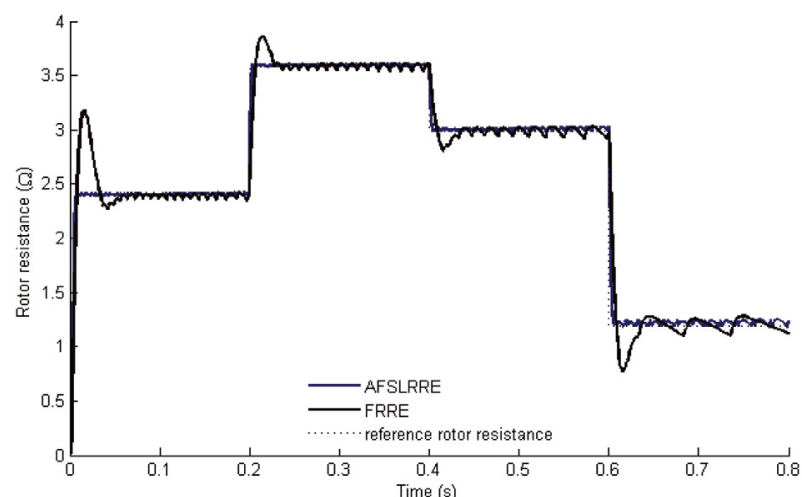

(a)

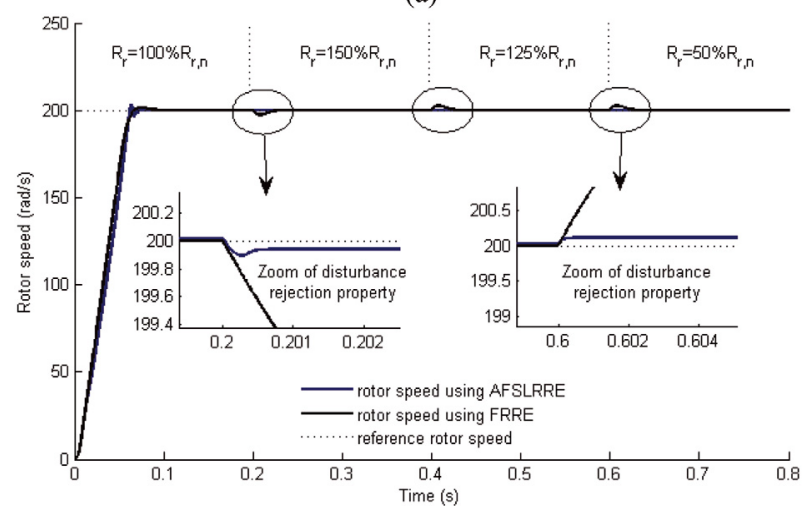

(b)

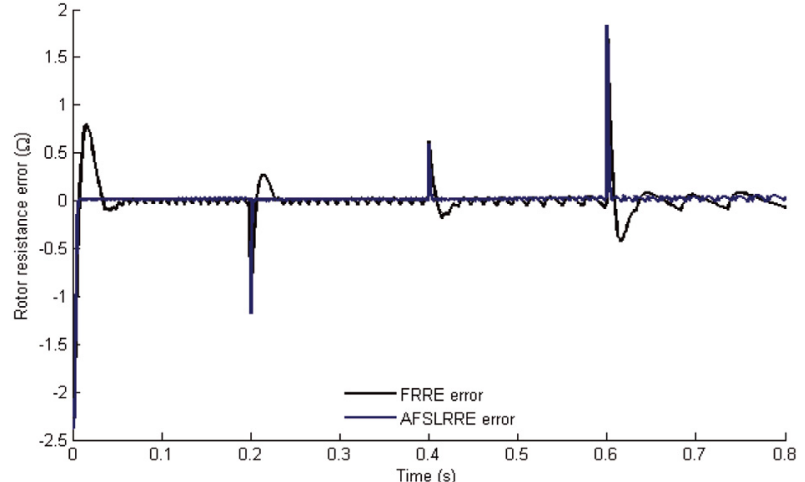

(a')

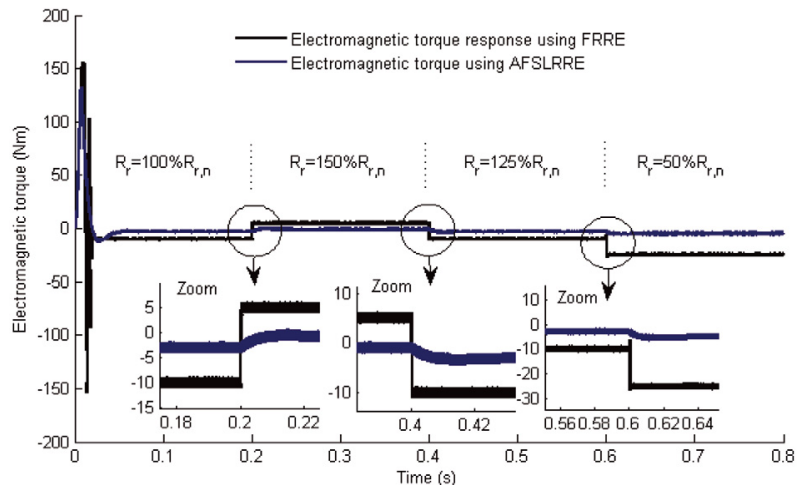

(c)

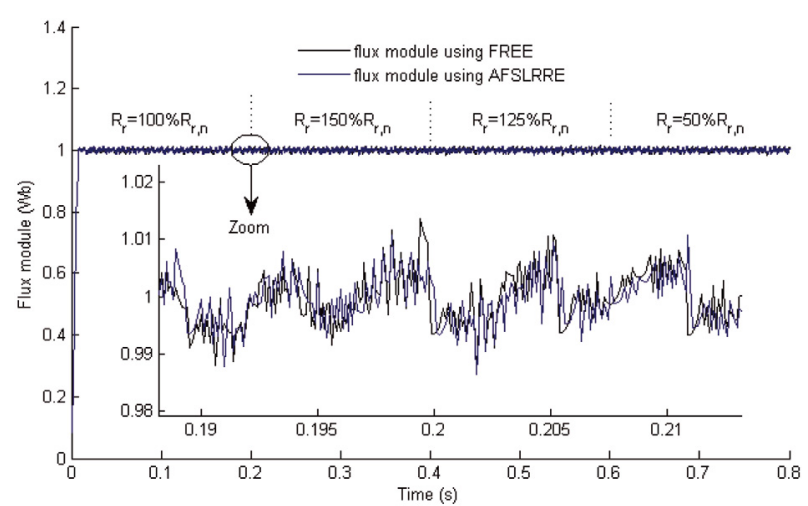

(d)

Fig. 5. AFSLRRE compared to FRRE. (a) rotor resistance estimation; (a') rotor resistance estimation error; (b) rotor speed; (c) electromagnetic torque; (d) flux module.

Table 2. Response of induction motor under different approaches proposed.

\begin{tabular}{|c|c|c|c|c|c|}
\hline Methods & Errors & $R_{r}=100 \% R_{r, n}$ & $R_{r}=150 \% R_{r, n}$ & $R_{r}=125 \% R_{r, n}$ & $R_{r}=50 \% R_{r, n}$ \\
\hline \multirow{4}{*}{ AFSLRRE } & Rotor resistance error & $0.01 \%$ & $0.03 \%$ & $0.02 \%$ & $0.05 \%$ \\
\hline & Rotor speed error & $0.002 \%$ & $0.003 \%$ & $0.005 \%$ & $0.009 \%$ \\
\hline & Flux module error & $0.12 \%$ & $0.12 \%$ & $0.12 \%$ & $0.12 \%$ \\
\hline & Electromagnetic torque error & $1.22 \%$ & $1.33 \%$ & $1.31 \%$ & $1.34 \%$ \\
\hline \multirow{4}{*}{ FRRE } & Rotor resistance error & $0.23 \%$ & $0.11 \%$ & $0.23 \%$ & $0.31 \%$ \\
\hline & Rotor speed error & $0.01 \%$ & $0.02 \%$ & $0.01 \%$ & $0.05 \%$ \\
\hline & Flux module error & $0.22 \%$ & $0.22 \%$ & $0.22 \%$ & $0.22 \%$ \\
\hline & Electromagnetic torque error & $2.2 \%$ & $2.5 \%$ & $2.3 \%$ & $2.9 \%$ \\
\hline
\end{tabular}


Table 3. The comparison of the computation time for both methods.

\begin{tabular}{cc}
\hline Methods & Execution time in Matlab \\
\hline AFSLRRE & $6.812 \mathrm{~min}$ \\
FRRE & $14.339 \mathrm{~min}$ \\
\hline
\end{tabular}

in literature. The optimal control vector is then obtained and the torque/current is kept at the maximum value corresponding to a given load torque.

\section{References}

[1] X. Yu, M.W. Dunnigan, B.W. Williams, A novel rotor resistance identification method for an indirect rotor fluxOriented controlled induction motor drive system, IEEE Trans. Power Electron. 17 (2005) 353-364

[2] L.A. Zadeh, Fuzzy sets, Inform. Control 8 (1965) 338-353

[3] L.A. Zadeh, Fuzzy logic, IEEE Comput. Mag. 21 (1988) 83-92

[4] R. Belohlavek, G.J. Klir, Concepts and fuzzy logic, Ph.D. dissertation, Massachusetts Institute of Technology, 2011

[5] G.J. Klir, B. Yuan, Fuzzy Sets and Fuzzy Logic: Theory and Applications, Prentice Hall, New Jersey, 1995

[6] L.X. Wang, Adaptive Fuzzy Systems and Control: Design and Stability Analysis, Prentice-Hall, New Jersey, 1994

[7] H. Kang, G. Vachtsevanos, Adaptive fuzzy logic control, IEEE International Conference on Fuzzy Systems, San Diego, CA, 1992, pp. 407-414

[8] S. Sastry, M. Bodson, Adaptive Control: Stability, Convergence and Robustness, Dover Publications Inc, 2011
[9] D.S. Reay, M.W. Dunnigan, Learning issues in model reference based fuzzy control, IEEE Proc. Control Theory Appl. 144 (1997) 605-611

[10] M. Russo, C.J. Lakhmi, Fuzzy Learning and Applications, CRC Press, 2001

[11] M.J. Jacobs, Self-learning Artificial Neural Networks, Randse Afrikaanse Universiteit, 1993

[12] E.M. Mamdani, S. Assilian, An experiment in linguistic synthesis with a fuzzy logic controller, Int. J. ManMachine Stud. 7 (1975) 1-13

[13] A. Trzynadlowski, Control of Induction Motors, Academic Press, 2000

[14] W. Leonhard, Control of Electrical Drives, 3nd edition, Springer, 2001

[15] P.C. Krause, O. Wasynczuk, S.D. Sudhoff, Analysis of Electric Machinery and Drive Systems, Wiley Interscience, New york, 2002

[16] B. Karanayil, M.F. Rahman, C. Grantham, Online stator and rotor resistance estimation scheme using artificial neural networks for vector controlled speed sensorless induction motor drive, IEEE Trans. Ind. Electron. 54 (2007) 167-176

[17] B. Karanayil, M.F. Rahman, C. Grantham, Stator and rotor resistance observers for induction motor drive using fuzzy logic and artificial neural networks, IEEE Trans. Energy Conversion 20 (2005) 771-780

[18] G. Kenné, T. Ahmed-Ali, F. Lamnabhi-Lagarrigue, A. Arzandé, J. Claude Vannier, An improved rotor resistance estimator for induction motors adaptive control, Electr. Power Syst. Res. 81 (2011) 930-941

[19] P. Roncero-Sanchez, A. Garcia-Cerraba, V. Feliu-Batlle, Rotor resistance estimation for induction machines with indirect field-orientation, Control Eng. Practice 15 (2007) 1119-1133 